\title{
Natural Language Processing: An Approach to Aid Emergency Services in COVID-19 Pandemic
}

\author{
Komal, Akshay Sharma
}

\begin{abstract}
In the recent years, Natural Language Processing (NLP) has been widely adopted in numerous applications to organize and structure knowledge to accomplish tasks like translation, summarization, named entity recognition, relationship extraction and speech recognition. With the advent of deep learning techniques, there has been significant increase in the processing efficiency of NLP based systems. The COVID-19 pandemic situation has challenged the medical research, IT operations, business processes and world economy at large. Numerous solutions are being developed to make lockdown and social distancing successful without causing much inconvenience to public. The current trends and applications of NLP can act as a critical support system for fighting COVID-19 pandemic situation. This paper presents a comparative assessment of various deep learning models for NLP techniques and proposes an NLP framework to develop essential and emergency services support system to minimize human interactions.
\end{abstract}

KEYWORDS- NLP, NLG, deep learning, artificial intelligence, name entity recognition, machine translation, COVID-19.

\section{INTRODUCTION}

COVID-19 has been declared as pandemic by WHO (World Health Organization) all across the world, owing to its rate of transmission and severe health issues among the infected people. Statistics clearly show that the infected people have surpassed 4.89 million $323 \mathrm{k}$ deaths and $1.69 \mathrm{M}$ recovered but still counting. Covid-19 primary transmits trough saliva of infected patient when they sneeze or touch an infected surface or object. The best solution that has been uncovered to contain COVID-19 is to make social distancing. Most of the countries have declared lockdown nationwide to reduce the rate of transmission among people but a bigger challenge is to balance the economy and provide support to citizens, for essential and emergency services while sitting in their homes.

\section{Manuscript received May 22, 2020}

Komal, Assistant Professor, Department of Computer Science and Engineering, Amity University Haryana, Gurugram, India (email: komal.sang@gmail.com)

Akshay Sharma, Student, Department of Computer Application, Amity University, Haryana, Gurugram, India
Numerous applications have been developed to help this process and many researchers have been working to find solutions in this direction. Natural Language Processing (NLP) techniques can be utilized to help illiterate people in the society where local language communication can be interpreted by the system and their requirements can be communicated and fulfilled by the channels available. NLP (Natural Language Processing) is a field of computer science that interacts with AI (Artificial Intelligence) models and linguistics [1]. NLP is used for understanding natural language of humans and process this input for various different applications using a variety of algorithms [1]-[9]. Various Machine Learning training algorithms study millions of examples of text words, sentences, and paragraphs written by humans [10]. By studying the samples, the training algorithms gain an understanding of the "context" of human speech, writing, and other modes of communication. In NLP, there are two components NLG (Natural language generation) and NLU (Natural Language Understanding) [1]. In NLG, it generates text and speech synthesis to generate spoken output. In NLU, it extracts information from and speech recognition to get information from speech.

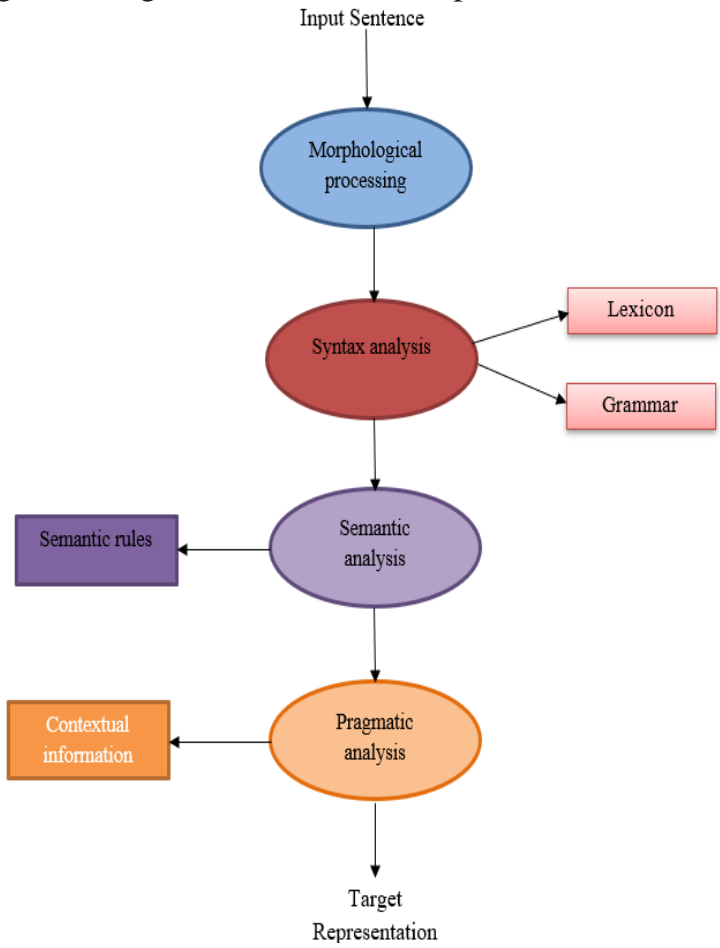

Fig 1: Steps used in Natural language processing [2]

As shown in above diagram, the first step is morphological process and it is concerned with the interplay between words and their relationship with other 
words [2]. In syntax analysis, the grammatical structure of sentence is found out. The semantic analysis phase is used to convey the meaning of words and group of words through rules and in last step i.e. pragmatic analysis

explains the context of spoken expressions. The paper is structured as follows: Section 2 discusses the widely used applications of NLP and prominently used AI models to support each application.

Section 3 presents the NLP based support system framework to aid emergency services as well as essential services in pandemic situation like covid-19. Section 4 concludes the paper with scope of future development.

\section{DEEP LEARNING MODELS FOR NLP APPLICATIONS}

Using Deep learning models, we can do better in translation, question answering, summarization, natural language inference etc. it seems good to work on deep learning but to do it effectively we need to have millions of labeled data. do it effectively we need to have millions of labeled data. The efficiency of these algorithms differs significantly in various NLP processes like POS tagging, parsing, named entity recognition, semantic role labeling, machine translation, dialogue systems and contextual embedding [3]. The analysis of various deep learning models and its applicability to NLP domain is presented in Table 1.

Table 1: Comparative assessment of Deep Learning Models for NLP processes [4] [5] [6] [7] [8]

\begin{tabular}{|c|c|c|c|}
\hline $\begin{array}{l}\text { Deep Learning } \\
\text { Models }\end{array}$ & Approach & $\begin{array}{l}\text { Model } \\
\text { Variants }\end{array}$ & $\begin{array}{l}\text { NLP } \\
\text { application } \\
\text { areas }\end{array}$ \\
\hline $\begin{array}{l}\text { Convolutional } \\
\text { Neural } \\
\text { Networks }\end{array}$ & $\begin{array}{l}\text { Extract } \\
\text { higher-level } \\
\text { features using } \\
\text { specialized } \\
\text { filters. }\end{array}$ & $\begin{array}{l}\text { TDNN } \\
\text { and } \\
\text { DCNN }\end{array}$ & $\begin{array}{l}\text {-Short-text } \\
\text { classification. } \\
\text {-Speech } \\
\text { recognition. } \\
\text {-Sentiment } \\
\text { analysis. }\end{array}$ \\
\hline $\begin{array}{l}\text { Recurrent } \\
\text { Neural } \\
\text { Networks }\end{array}$ & $\begin{array}{l}\text { Find context } \\
\text { dependencies } \\
\text { in sequential } \\
\text { information of } \\
\text { arbitrary } \\
\text { length }\end{array}$ & $\begin{array}{l}\text { LSTM, } \\
\text { ResNets } \\
\text { and } \\
\text { GRU }\end{array}$ & $\begin{array}{l}\text {-Word \& } \\
\text { sentence } \\
\text { classification. } \\
\text {-Semantic } \\
\text { matching. } \\
\text {-Machine } \\
\text { Translation. }\end{array}$ \\
\hline $\begin{array}{l}\text { Recursive } \\
\text { Neural } \\
\text { Networks }\end{array}$ & $\begin{array}{l}\text { Combine } \\
\text { constituents in } \\
\text { a bottom-up } \\
\text { approach to } \\
\text { calculate } \\
\text { higher-level } \\
\text { information. }\end{array}$ & $\begin{array}{l}\text { MN- } \\
\text { RNN } \\
\text { and } \\
\text { RNTN }\end{array}$ & $\begin{array}{l}\text {-Parsing. } \\
\text {-Sentiment } \\
\text { Analysis. }\end{array}$ \\
\hline $\begin{array}{l}\text { Deep } \\
\text { Generative } \\
\text { models }\end{array}$ & $\begin{array}{l}\text { Generate } \\
\text { sample } \\
\text { outputs from } \\
\text { discrete input } \\
\text { gathered from } \\
\text { latent space. }\end{array}$ & $\begin{array}{l}\text { VAEs } \\
\text { and } \\
\text { GANs }\end{array}$ & $\begin{array}{l}\text {-Text } \\
\text { Generations }\end{array}$ \\
\hline
\end{tabular}

\section{PROPOSED FRAMEWORK FOR COVID-19 PANDEMIC SUPPORT SYSTEM}

This section presents theoretical framework based on NLP to aid support system and services by taking Voice-based requests in different languages, process information, derive results and provide voice-based output as depicted in fig. 2.

\section{A. Methodology to aid doctors \& medical staff}

It is crucial for medical staff- nurses, doctors and other supporting staff to be safe and thus, we devised an approach with the help of which doctors can refrain from touching objects like medical records and visiting patients frequently for gaining symptomatic information and executing treatments. This framework (as shown in fig. 2) can help doctors to fetch information from database of medical reports and provide guidelines to quarantined patients and/or nurses and other medical staff on further treatment. In the first step, speech recognition module breaks down the speech recording into individual sound, analyzing each sound, and converts it into text by using acoustic model, decoder, language algorithm and lexicon of the language. Using second and third step, sentences are converted into tokens. These tokens are then analyzed for grammatical corrections in fourth step (text lemmatization).

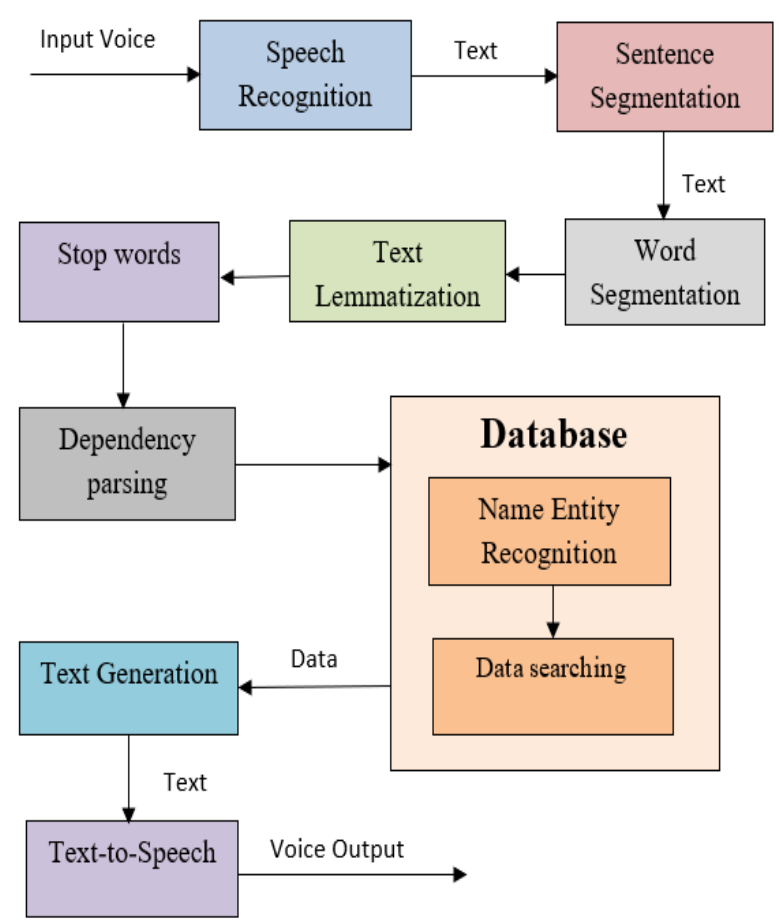

Fig 2: NLP Framework to support medical staff 
International Journal of Innovative Research in Computer Science \& Technology (IJIRCST) ISSN: 2347-5552, Volume-8, Issue-3, May 2020 https://doi.org/10.21276/ijircst.2020.8.3.32 www.ijircst.org

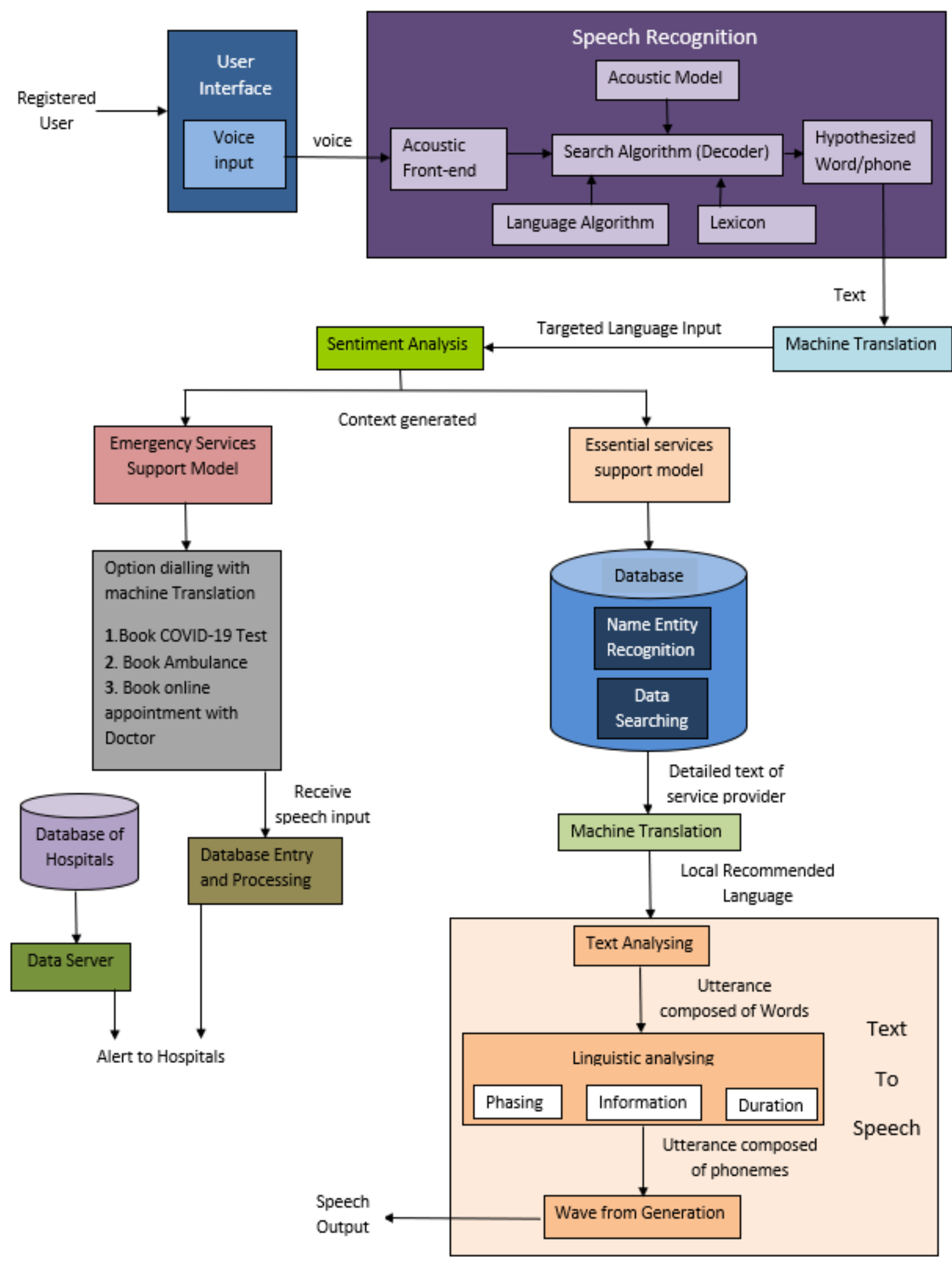

Fig 3: Framework to aid support system for Emergency and essential services 
Using stop words, we break information into components and perform database search using identified keywords in name entity recognition phase. Based on searched information in database, text generation module tries to complete or frame the sentence by using some algorithms. It automatically generates natural language text which can further be converted to voice output using text to speech synthesis model.

\section{B. Methodology to aid citizens}

The proposed methodology is presented in fig. 3 where a user needs to register its details including name, current location and contact number before using the system. Once the user is registered, he/she can use user Interface to request information, services and process commands using voice input (in any supported language). The speech recognition module converts the source language speech into source language text which undergoes machine translation phase, where the source text is converted into targeted language test (i.e. English). The output generated by this phase is analyzed for contextual findings (critical emergency or general-purpose need) through sentiment analysis based model and corresponding module is called up internally. The Emergency Service Support Module (EmSSM) provides three voice dialing options (as shown in fig.3) and action is performed based on input received with an alert raised to nearby hospital. On the other hand, Essential Service Support Module (EsSSM) performs named entity recognition on database to fetch results, generating text and transforming it into local language text using machine translation. Corresponding language acoustic model can then be used to provide voice-based results.

\section{CONCLUSION}

The paper analyzed deep learning models for NLP applications and suggested best-suited approaches. The Framework proposed in the paper specifically targets to overcome the technical and language barrier problems of illiterate people of India including migrant laborers. The system takes voice input with speech processing system having multiple regional language support. The intelligent deep learning models can thus discover the context and process information to provide solutions in the regional language itself without the need or intervention of any human interaction. Even doctors can be benefitted through the NLP framework to check reports and provide treatment guidelines to patients without meeting them in person.

\section{REFERENCES}

[1] Diksha Khurana, Aditya Koli, Kiran Khatter and Sukhdev Singh "Natural Language Processing: State of The Art, Current Trends and Challenges" Avilable: https://arxiv.org/ftp/arxiv/papers/1708/1708.0514 8.pdf

[2] Selvapandian, R. Udaya Nirmala Mary, C. Karthikeyan "Artificial Intelligence In Online Shopping Using Natural Language Processing (NLP)" Journal of Critical Reviews ISSN- 2394-5125 Vol 7,
Issue 4, 2020

[3] Young, T., Hazarika, D., Poria, S., \& Cambria, E. (2018). Recent Trends in Deep Learning Based Natural Language Processing [Review Article]. IEEE Computational Intelligence Magazine, 13(3), 55-75.

[4] Ossama Abdel-Hamid, Abdel-rahman Mohamed, Hui Jiang, Li Deng, Gerald Penn, and Dong Yu" Convolutional Neural Networks for Speech Recognition" IEEE/ACM Transactions On Audio, Speech, And Language Processing, Vol. 22, No. 10, October 2014

[5] Iqbal, T.,Qureshi, S., "The Survey: Text Generation Models in Deep Learning", Journal of King Saud University - Computer and Information Sciences (2020), doi: https://doi.org/10.1016/j.jksuci. 2020.04.001

[6] Zheng Wang and Qingbiao Wu "An Integrated Deep Generative Model for Text Classification and Generation" Volume 2018 |Article ID 7529286 | 8 pages | https://doi.org/10.1155/2018/75 29286

[7] Kiran Baktha, , and B K Tripathy, Senior Member, IEEE "Investigation of Recurrent Neural Networks in the field of Sentiment Analysis" International Conference on Communication and Signal Processing, April 6-8, 2017, India

[8] Boxuan Yue ID, Junwei Fu and Jun Liang "Residual Recurrent Neural Networks for Learning Sequential Representations" Received: 1 February 2018; Accepted: 4 March 2018; Published: 6 March 2018 doi:10.3390/info9030056

[9] Prakash M Nadkarni, Lucila Ohno-Machado, Wendy W Chapman "Natural language processing: an introduction" Journal of the American Medical Informatics Association, Volume 18, Issue 5, September 2011, Pages 544551, https://doi.org/10.1136/amiajnl-2011-000464

[10] Surbhi, M. C. (2013). Natural language processing future. 2013 International Conference on Optical Imaging Sensor and Security Issue 3 / July/2013 (ICOSSdoi:10.1109/icoiss.2013.66784

\section{ABOUT THE AUTHORS}

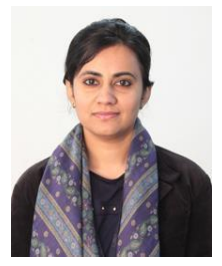

Komal has done B.E. in Information Technology from Maharshi Dayanand University, Rohtak and M.Tech in Computer Science \& Engineering from Kurukshetra University. She is currently pursuing Ph.D. and currently working as Assistant Professor in Department of Computer Science and Engineering, Amity University Haryana, Gurugram, India. She is a member of IET U.K, IACSIT, IAENG and UACEE,.She has authored 2 books and published more than 16 research papers in reputed international journals including Scopus indexed. Her main research work 


\section{International Journal of Innovative Research in Computer Science \& Technology (IJIRCST)}

ISSN: 2347-5552, Volume-8, Issue-3, May 2020

https://doi.org/10.21276/ijircst.2020.8.3.32

www.ijircst.org

focuses on Deep Learning, Network security, Cloud computing and security, IoT. She has 8 years of teaching experience and Research Experience.

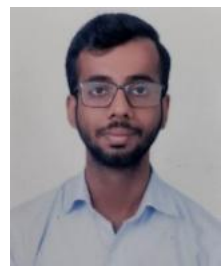

Akshay Sharma has done his Bachelor of Computer Application (BCA) and currently persuing Master of Computer Application (MCA) from Amity University Haryana, Gurugram, India 